\title{
Temporal properties of Swift GRBs with known redshifts
}

\author{
Roger Edwin O'Connor* $\dagger$ \\ School of Physics, University College Dublin, Dublin 4, Ireland. \\ E-mail: roger.oconnoradcenr.1e
}

\section{Sheila McBreen, Lorraine Hanlon, Antonio Martin-Carrillo, Brian McBreen}

School of Physics, University College Dublin, Dublin 4, Ireland.

\section{Suzanne Foley}

Max-Planck-Institut für extraterrestrische Physik, Giessenbachstraße, 85748 Garching, Germany.

The Swift Gamma Ray Burst (GRB) mission has enabled the rapid detection of GRBs and the determination of the redshift of a greater proportion of source counterparts than previous missions. The mean redshift of the Swift GRBs is significantly higher than that of earlier missions.

We present the results of a temporal analysis performed on 134 Swift long GRBs with known redshift. $\ddagger$

The GRBs were denoised using wavelets and subjected to an automatic pulse selection algorithm with the objective of identifying pulses. The rise times, fall times, full-width at half-maxima (FWHM), pulse amplitudes and pulse areas were measured and the frequency distributions of some of them are presented here.

The results provide a comprehensive description of the pulse profiles in Swift GRBs with known redshift, determining that the temporal properties are consistent with lognormal distributions and that correcting for the known redshift of the source does not have a significant impact on the distributions. Lognormal distributions arise from random multiplicative processes and abound in a variety of natural phenomena.

8th INTEGRAL Workshop "The Restless Gamma-ray Universe"

September 27-30 2010

Dublin Castle, Dublin, Ireland

\footnotetext{
*Speaker.

${ }^{\dagger}$ With thanks to the Department of Communications, Energy and Natural Resources, Ireland.

\#This research has made use of data obtained from the High Energy Astrophysics Science Archive Research Center (HEASARC), provided by NASA's Goddard Space Flight Center.
} 


\section{Introduction}

Gamma-ray bursts (GRBs) are the most powerful events known, in which $\approx 10^{51} \mathrm{ergs}$ is released in only $0.01-1000$ seconds in gamma-rays and the time profiles display rapid variability. The most popular model of the central engine is a hyper-accreting black hole, in which the energy of the relativistic jets, that make the gamma-ray emission, is produced by the accretion of a massive disk onto a newly created black hole. In the internal shock model, the observed time profile tracks the activity of the central engine. For a recent review of GRBs see $[1 ; 2]$.

The work presented here expands on an earlier analysis of the time profiles of BATSE GRBs [3; 4]. However only a few BATSE GRBs had a known redshift [3]. Since the redshift is known for a significant proportion of Swift GRBs, this enabled the relationship between redshift and the temporal properties of the light curves to be more fully examined. The Swift bursts are part of a deeper survey than BATSE and the redshifts of the long-duration Swift bursts, ( $\mathrm{T}_{90} \gtrsim 2 \mathrm{~s}$ ), are statistically larger than pre-Swift missions with a mean of $\approx 2.2$ for this sample compared to $\sim 1$ for pre-Swift bursts [5]. This provided the opportunity to examine the properties of the light curves of GRBs over a wide range of redshifts and to yield further insight into the processes which give rise to GRBs.

\section{Method}

The time profiles of 134 long Swift GRBs with known redshift were analysed using the method given in $[3 ; 4]$. All four Swift channels were added together to maximise the signal to noise ratio. The light curves were de-noised using 1-D Stationary Wavelet Transform De-noising from the MATLAB wavelet toolbox. The parameters, including the wavelet filter, level, noise structure, thresholding method and settings were adjusted to give the best fit between the de-noised signal and raw data. Once the denoised signal had been determined, the pulse selection algorithm automatically selected pulses based on objective criteria [3]. The algorithm therefore minimised the subjective element of pulse selection.

Peak recognition and decomposition software was adapted to extract the pulse properties including rise times, fall times, full-width at half-maxima (FWHM), pulse amplitudes and pulse areas of the Swift GRBs with known redshift. The pulse properties were extracted for a range of significance and separation from adjacent pulses. The pulse properties were corrected from the known redshifts of the sources.

The effect of neighbouring pulses was considered using two parameters: a significance threshold and an isolation threshold. The significance threshold, $\tau_{\sigma}$, determines the amount by which the local maximum and minimum of a pulse must be separated in order for a pulse to be selected, while the isolation threshold, $\tau_{i}$, determines the amount by which the local maximum of a pulse must be greater than the highest local minimum. This method has the benefit of making the process of selecting the pulses more objective than a purely visual selection. The algorithm selects pulses with $5 \sigma$ above the background noise level $\left(\tau_{\sigma} \geq 5\right)$ and a threshold of not less than $50 \%$ isolation from neighbouring pulses $\left(\tau_{i} \geq 50 \%\right)$. From the sample of 134 long-duration GRBs, a total of 371 pulses were selected with $\tau_{\sigma} \geq 5$ and of these 257 had $\tau_{i} \geq 50 \%$. For the selected pulse within a burst the algorithm determined $\mathrm{T}_{\text {rise }}, \mathrm{T}_{\text {fall }}, \mathrm{FWHM}$ and time intervals, $\mathrm{T}_{\text {int }}$. In addition to these 


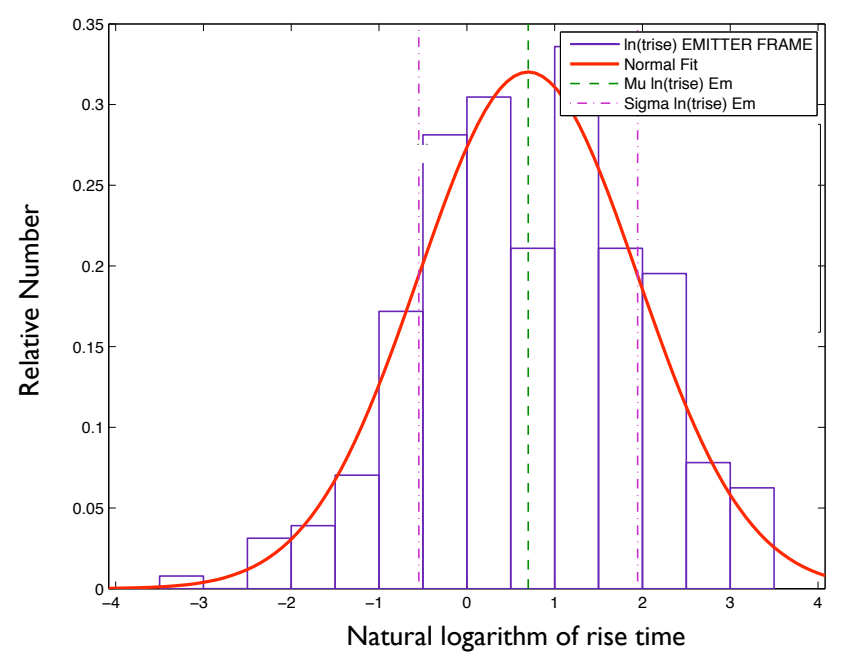

Figure 1: The frequency distribution of the rise time ( $\mathrm{t}_{\text {rise }}$ ) of the pulses with $\tau_{\sigma} \geq 5$ and $\tau_{i} \geq 50 \%$. The solid continuous curve (red) is the lognormal fit of the data.

temporal properties the algorithm also determined the maximum amplitude of the pulse, $C_{\text {max }}$, and the area of the pulse.

The time intervals between pulses were also analysed. All pulses with $\tau_{\sigma} \geq 5$ were analysed for the time intervals, not just the isolated pulses. All of the long-duration Swift GRBs of known redshift were analysed from GRB 050126 to GRB 091208B with the exception of 6 GRBs for which BAT was not data available, 1 for which the data was unusable and 2 which were so weak that the automated algorithm was unable to analyse them. This resulted in 134 bright and weak long-duration GRBs being analysed with BAT fluence $(15-150 \mathrm{keV})$ ranging from a minimum of $0.68 \times 10^{-7} \mathrm{erg} \mathrm{cm}^{-2}$ (GRB 050406) to a maximum of $1.05 \times 10^{-7} \mathrm{erg} \mathrm{cm}^{-2}$ (GRB 090618).

The MATLAB Statistics Toolbox was used to fit a normal distribution to the natural logarithm of the data sample. To fit the distribution MATLAB used the sample data to calculate the maximum likelihood estimates of the parameters of the normal distribution. Maximum Likelihood Estimation (MLE) computed the estimates by numerically maximizing the distribution's log-likelihood. The larger the value of the log-likelihood the better the fit. Because the distribution from which the sample data came was unknown it was not possible to accurately determine the unknown parameters of that distribution from the sample data. Therefore several statistical tests were carried out to determine whether the data came from a family of distributions. The $95 \%$ confidence intervals for the estimated parameters were calculated. Not only was it possible to estimate the parameters of the normal distribution using MLE, but it was also possible to guarantee with $95 \%$ confidence that the true unknown parameters of the distribution belong to these confidence intervals.

\section{Results}

The frequency distributions of the rise time, fall time and FWHM are presented in Fig. [, $\square$ 


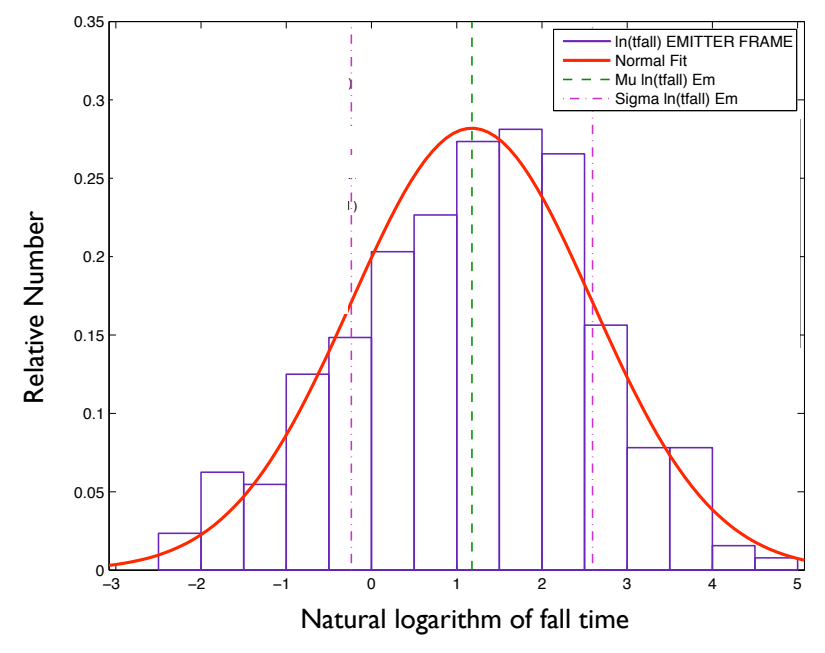

Figure 2: The frequency distribution of the fall time ( $\left.\mathrm{t}_{\text {fall }}\right)$ of the pulses with $\tau_{\sigma} \geq 5$ and $\tau_{i} \geq 50 \%$. The solid continuous curve (red) is the lognormal fit of the data.

and $\square$ respectively. Fig. 3 and $⿴ 囗 十$ show the rise time and fall time data plotted as a cumulative fraction such that a lognormal distribution yields a straight line [6].

The geometric mean (median) values of the pulse properties of the 134 long GRBs of known redshift in the observer and emitter frames are given in Table $\mathbf{m}$.

(a) Observer Frame

\begin{tabular}{|l|c|}
\hline Pulse Property & Median \\
\hline Rise Time: $\mathrm{T}_{\text {rise }}(\mathrm{s})$ & 6.1 \\
Fall Time: $\mathrm{T}_{\text {fall }}(\mathrm{s})$ & 9.9 \\
FWHM (s) & 6.8 \\
Time Interval: $\mathrm{T}_{\text {int }}(\mathrm{s})$ & 11.0 \\
Pulse Amplitude: $\mathrm{C}_{\max }$ (count rate) & 2399.9 \\
Pulse Area: (counts) & 38357.4 \\
\hline
\end{tabular}

(b) Emitter Frame

\begin{tabular}{|l|c|}
\hline Pulse Property & Median \\
\hline Rise Time: $\mathrm{T}_{\text {rise }}(\mathrm{s})$ & 2.0 \\
Fall Time: $\mathrm{T}_{\text {fall }}(\mathrm{s})$ & 3.2 \\
FWHM (s) & 2.2 \\
Time Interval: $\mathrm{T}_{\text {int }}(\mathrm{s})$ & 3.8 \\
\hline
\end{tabular}

Table 1: The geometric mean (median) values of the pulse properties of the 134 long Swift GRBs of known redshift in the observer and emitter frames.

\section{Discussion}

The results support the concept that the frequency distributions of the rise times, fall times, FWHM, pulse amplitudes and pulse areas are all consistent with lognormal distributions provided the pulses are well separated.

The lognormal distribution arises from a statistical process whose result depends on a product of probabilities arising from a combination of independent events. It therefore identifies the statistical process but not the combination of events that lead to the formation of pulses. Random multiplicative processes abound in a variety of natural phenomena and a good example is the statistical 


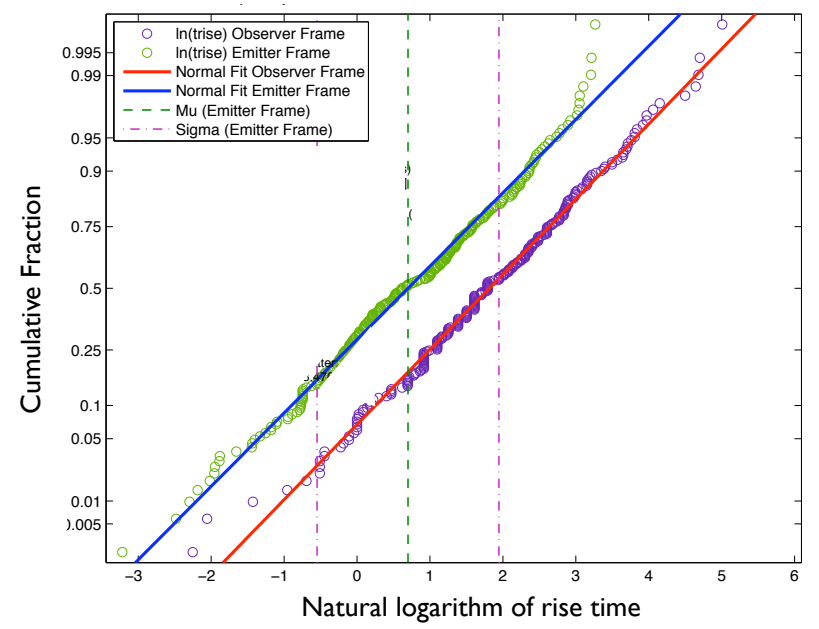

Figure 3: The rise time data given in Fig. 1 (purple and green) are plotted as a cumulative fraction such that a lognormal distribution yields a straight line for the observer (red) and emitter frames (blue).

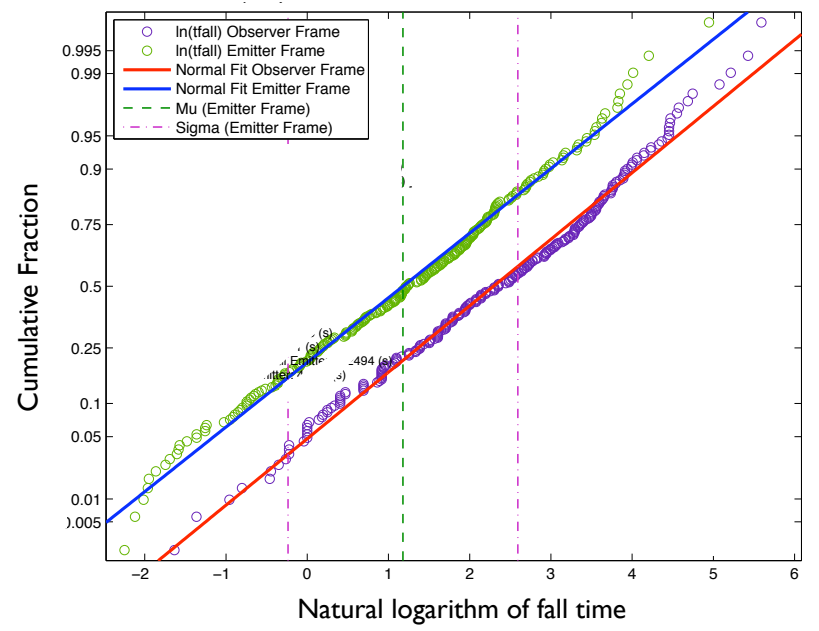

Figure 4: The fall time data given in Fig. 2 (purple and green) are plotted as a cumulative fraction such that a lognormal distribution yields a straight line for the observer (red) and emitter frames (blue). 


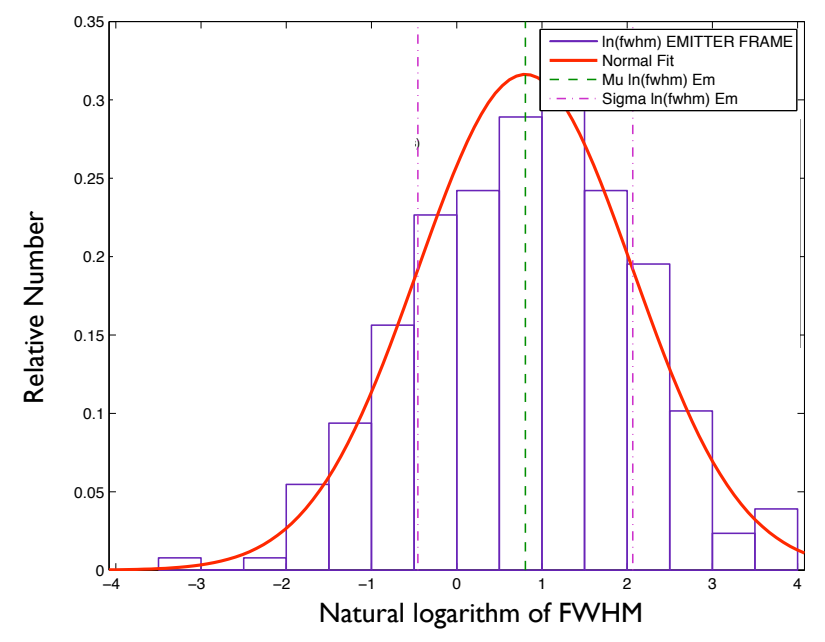

Figure 5: The frequency distribution of the full width at half maximum (FWHM) of the pulses with $\tau_{\sigma} \geq 5$ and $\tau_{i} \geq 50 \%$

properties of strokes in flashes of lightning [3].

Correcting for the known redshift of the source does not cause significant changes in the lognormal distributions of the pulse properties. This shows that the independent events involved in the generation of the lognormal distribution are the dominant controlling factors and not the redshift. This also shows that the redshift cannot be obtained from the pulse properties of GRBs. A full report of these results will be presented elsewhere (O'Connor et al. in preparation).

\section{References}

[1] G. Vedrenne and J.-L. Atteia. Gamma-Ray Bursts. Springer Berlin Heidelberg, 2009.

[2] B. Zhang. Open Questions in GRB Physics. Arxiv preprint astro-ph/1 104.0932, 2011.

[3] F. Quilligan, B. McBreen, L. Hanlon, S. McBreen, K. J. Hurley, and D. Watson. Temporal properties of gamma ray bursts as signatures of jets from the central engine. Astronomy and Astrophysics, 385:377-398, 2002.

[4] S. McBreen, F. Quilligan, B. McBreen, L. Hanlon, and D. Watson. Temporal properties of the short gamma-ray bursts. Astronomy and Astrophysics, 380:L31-L34, 2001.

[5] Y.Q. Lin. Comparison between Swift and pre-Swift gamma-ray bursts. Research in Astronomy and Astrophysics, 9:682, 2009.

[6] J. Aitchison and J. A. C. Brown. The Lognormal Distribution. Cambridge University Press, 1957. 